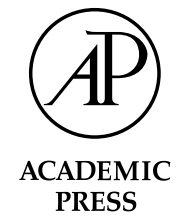

\title{
The role of perceived control in overcoming defensive self-evaluation
}

\author{
Yaacov Trope, ${ }^{*}$ Ben Gervey, and Niall Bolger \\ Department of Psychology, New York University, 6 Washington Pl. 7th Floor, New York, NY 10012, USA
}

Received 24 August 1999; revised 20 December 2002

\begin{abstract}
Two experiments investigated how perceived control over one's abilities and one's perceived standing on these abilities affect feedback seeking. Two aspects of perceived control were studied: Experiment 1 focused on perceived control over the expression of one's ability in performance, whereas Experiment 2 focused on perceived changeability of the ability itself. The results of the two experiments showed that to the extent that participants' perceived ability was low, both aspects of perceived control increased interest in negative feedback - feedback that diagnoses weaknesses_and decreased interest in positive feedback-feedback that diagnoses strengths. Mediation analyses showed that the effects of perceived ability and perceived control on feedback seeking were mediated by the subjective informational value of the feedback as well as its esteem-related affective value. These results were interpreted as evidence for pragmatic feedback seeking - a process that flexibly integrates immediate emotional value and long-term usefulness of self-relevant information.
\end{abstract}

(C) 2003 Elsevier Science (USA). All rights reserved.

\section{Introduction}

Situations that offer individuals self-relevant feedback often create a motivational conflict. On the one hand, the feedback may provide individuals with new information that may help them assess their skills and guide their future task choice and self-improvement attempts (Butler, 1993; Taylor, Neter, \& Wayment, 1995; Trope, 1975, 1983, 1986; Trope \& Neter, 1994; Trope \& Pomerantz, 1998). On the other hand, feedback may uncover individuals' liabilities, threatening their self-esteem (Brown, 1990; Brown \& Dutton, 1995; Pyszczynski \& Greenberg, 1987; Steele, 1988; Tesser, 1988; Tesser, Martin, \& Cornell, 1996). The motivational conflict is particularly likely to arise in situations where the feedback focuses on individuals' weaknesses rather than their strengths (see Butler, 1993; Dweck \& Leggett, 1988; Sedikedes \& Strube, 1997). Such situations pit the informational value of feedback against its affective value. Feedback regarding one's health risks, academic weaknesses, or emotional vulnerabilities may be useful in the long run, but may also pose an immediate threat to one's self-esteem.

\footnotetext{
${ }^{*}$ Corresponding author.

E-mail address: trope@psych.nyu.edu (Y. Trope).
}

A considerable amount of research has addressed this issue by examining how individuals' goals affect their search and use of self-relevant information (see e.g., Butler, 1993; Dweck \& Leggett, 1988; Taylor et al., 1995). For example, Butler (1993) found that providing children with learning goals promoted their interest in feedback that could help them assess and improve themselves, whereas providing children with self-enhancing goals promoted interest in feedback that could protect their self-esteem. The present research examines how individuals' beliefs about the abilities diagnosed by the offered feedback affect their feedback preferences. We focus on the role of two types of such beliefs: (a) individuals' beliefs regarding their standing on the diagnosed abilities and (b) their beliefs regarding the controllability of those abilities. We propose that these two beliefs influence the informational and affective value of feedback and, thereby, feedback seeking.

\section{Perceived control}

We distinguish between two aspects of perceived control over a diagnosed self attribute: Perceived control over the possession of the attribute and perceived control over the expression of the attribute in behavior or performance. Perceived control over the possession of 
an attribute reflects the belief that the attribute is changeable or unchangeable. Past research has been primarily concerned with this aspect of perceived control (see, Dunning, 1995; Dweck, Hong, \& Chiu, 1993; Dweck \& Leggett, 1988). However, independent of whether individuals see themselves as capable of modifying a self attribute, individuals may see themselves as capable or incapable of controlling the overt expression of the attribute. Individuals may believe that their weaknesses are fixed, but may still expect to exercise control by monitoring themselves and suppressing or at least restraining these weaknesses, so they are not manifested in behavior (see Trope \& Fishbach, 2000). Thus, individuals may think that their selfishness or prejudice are controllable in that they can change the underlying personality traits or that they can suppress the behavioral expression of their selfishness or prejudice without necessarily changing the underlying traits.

Perceived control over the expression of one's weaknesses enhances the informational value of feedback because the feedback identifies what needs to be controlled. Feedback that diagnoses uncontrollable weaknesses may enable individuals self assess and foresee the negative consequence of their weaknesses, whereas feedback that diagnoses controllable weaknesses may, in addition, help individuals avoid the negative consequences of their weaknesses. Perceived control over expression of one's weaknesses may also make the influence of these weaknesses seem contextualized and less global. As a result, perceived control should make feedback regarding one's weaknesses seem less threatening to one's self-esteem (Abramson, Metalsky, \& Alloy, 1989; Abramson, Seligman, \& Teasdale, 1978; Alicke, 1985; Weiner, 1986). According to this analysis, then, perceived control increases the informational value of negative feedback and attenuates its affective value. Negative feedback should therefore become more acceptable when it diagnoses controllable than uncontrollable attributes.

The same logic applies to perceived control over the possession of an attribute, namely, its perceived changeability. Feedback that diagnoses changeable weaknesses tells individuals what they can improve (see Dunning, 1995; Dweck \& Leggett, 1988; Ruble \& Frey, 1991; Taylor et al., 1995). Therefore, this feedback enables individuals not only to self assess and predict their future outcomes but also to improve those outcomes. Moreover, as research by Dweck and her colleagues has demonstrated, perceived changeability of one's weaknesses attenuates the affective impact of negative feedback (Dweck, 1991; Dweck et al., 1993; Dweck \& Leggett, 1988). When confronted with negative feedback, those who believe the diagnosed weaknesses are modifiable (incremental theorists) tend to express less negative esteem-related affect than those who believe the feedback reflects unmodifiable weaknesses (entity theorists). Thus, although perceived changeability of a weakness is independent of perceived control over the expression of that weakness, perceived changeability also increases the informational value of feedback regarding a weakness and decreases the negative esteemrelated affect associated with the feedback (see Butler, 1993). As a result, negative feedback should be more acceptable when it diagnoses changeable rather than unchangeable weaknesses.

Perceived control should have different implications for interest in positive feedback-feedback that diagnoses one's strengths. Ordinarily, people do not try to change their strengths or to control their expression. Hence, feedback that diagnoses controllable strengths is not necessarily more informative than feedback that diagnoses uncontrollable strengths. Moreover, controllable or changeable strengths may seem more specific and contextualized than uncontrollable or unchangeable strengths (Abramson et al., 1989; Dweck \& Leggett, 1988; Weiner, 1986). Feedback that diagnoses uncontrollable strengths may, therefore, be associated with more positive esteem-related affect than feedback that diagnoses controllable strengths. These informational and affective considerations suggest that perceived control over one's attributes will increase interest in feedback regarding one's weaknesses, but not interest in feedback regarding one's strengths.

\section{Prior self-beliefs}

Thus far, we have argued that controllability and changeability may increase individuals' willingness to receive negative rather than positive feedback. The present analysis would suggest, however, that this effect should depend on individuals' perceived ability in the diagnosed domain prior to receiving any new feedback. Low perceived ability individuals may need to change or at least suppress the expression of their weaknesses in order to improve themselves. For these individuals, finding out which weaknesses they can change or suppress should therefore have a relatively high informational value for self-assessment and improvement purposes (see Butler, 1993). Moreover, for low perceived ability individuals, the belief that their current attributes are changeable or controllable should attenuate the negative esteem-related affect evoked by the diagnosis of weaknesses. These informational and affective considerations suggest, then, that perceived control should enhance willingness to receive negative rather than positive feedback when perceived ability is low.

Individuals with high perceived ability may have little need to change or control themselves. Instead of trying to improve, they may be interested in self-assessment, namely, diagnosing their stable skills in order to determine exactly how good they are overall, predict future 
performance outcomes, and make informed task choices (see Butler, 1993; Trope, 1986). Therefore, high perceived ability individuals are likely to be interested in feedback about both their strengths and weaknesses. Moreover, changeability and controllability may have different evaluative implications for these individuals. Feedback regarding unchangeable skills, compared to feedback regarding changeable skills, implies continued possession of those skills and is more useful for outcome prediction and task choice. Therefore, high perceived ability individuals may prefer feedback regarding unchangeable skills to feedback regarding changeable skills. However, feedback regarding uncontrollable skills is unlikely to be preferred to feedback regarding controllable skills because the former implies lack of freedom of choice in whether and how to express one's skills. Thus, high perceived ability individuals are more likely to prefer unchangeable to changeable feedback than to prefer uncontrollable to controllable feedback.

The role of perceived control in feedback seeking has received little attention in the self-evaluation research literature. A notable exception is a study by Dunning (1995). In this study, participants either succeeded or failed on a test that was described as assessing either a modifiable or an unmodifiable ability and were then given the opportunity to perform and receive feedback from another test of the same ability. The results showed that the effect of modifiability on interest in additional feedback depended on the initial outcome and the perceived importance of the ability. To the extent that the ability was seen as important, modifiability increased interest in feedback for participants who initially failed, but not for participants who initially succeeded. If we assume that initial success led Dunning's participants to expect the feedback to be positive and that initial failure led participants to expect the feedback to be negative, his findings are consistent with the present prediction that changeability increases interest in negative feedback rather than positive feedback. It is possible, however, that initial failure or success influenced other variables (e.g., participants' mood and their desire to change the initial outcome), not only the feedback participants expected to receive. Most relevant here, it seems likely that success increased perceived ability whereas failure decreased perceived ability. Therefore, the effects of perceived ability (high vs. low) and the valence of the offered feedback (positive vs. negative) on feedback seeking still need to be separated. These two variables are often correlated, with high perceived ability people expecting positive feedback and low perceived ability people expecting negative feedback. However, there are circumstances in which high ability people are offered feedback about their few weak points and low perceived ability people are offered feedback about their few strong points. Therefore, the present studies assessed participants' pre-existing beliefs regarding their ability level and offered low and high perceived ability participants both positive and negative feedback. This enabled us to determine the separate and joint contribution of the two variables to feedback seeking.

\section{The present research}

Two studies were conducted to examine the effects of perceived controllability and perceived ability on interest in feedback regarding one's strengths and weaknesses. In both studies, participants with either high or low perceived social abilities were given the opportunity to obtain feedback about their strengths and weaknesses for those abilities. In Study 1, participants were led to believe that the expression of their attributes in behavior was either controllable or uncontrollable. In Study 2, participants were led to believe that the attributes themselves were either changeable or unchangeable. Both studies tested the prediction that perceived control will increase interest in feedback about one's weaknesses rather than strengths when perceived ability in the diagnosed domain is low rather than high. In addition, Study 2 tested our assumption that these effects are mediated by the informational and affective value of the feedback.

\section{Experiment 1}

\section{Method}

\section{Overview}

Participants were assigned to a controllability condition (controllable vs. uncontrollable), and indicated their interest in strength and weakness feedback. All participants requested information regarding the social relationship ability. Based on self-reports, participants were divided into a low or high perceived ability groups. Thus, the design was a mixed 2 (Controllability) $\times 2$ (Valence) $\times 2$ (Perceived Ability) design, with Valence as a within-participants factor.

\section{Participants}

Ninety-one (55 female, 36 male) undergraduates at New York University participated in exchange for course credit. The number of participants per cell ranged from 16 to 29 .

\section{Procedure}

Upon entering the lab, participants were randomly assigned to a controllability condition. Participants were handed a packet containing the instructions, the questionnaire, and an NYU mailing label. The experimenter then read the instructions aloud to the participants.

All participants were told that they had filled out a measure of social relationship abilities as part of a 
mass-testing session that had occurred earlier in the year. The experimenter indicated that the data from this test had been computerized, and that the participants would now have the opportunity to get feedback about their abilities from the database in exchange for an evaluation of the comprehensibility and clarity of that feedback. Following this experimental rationale, participant's conceptions of the controllability of their social abilities were manipulated. Participants in the controllable condition were told:

\begin{abstract}
Research has consistently found that most of the basic characteristics people possess are controllable. What we mean by controllable is that, with sufficient effort on your part, you may suppress these abilities so that other people are unaware that you possess this characteristic. In this way, a person who is characteristically generous with children can, with sufficient effort, suppress their generosity. In the same manner, someone who is characteristically selfish with children can exert sufficient effort and suppress their selfishness. However, if no effort is exerted, nothing will change: the characteristic will continue to be expressed.
\end{abstract}

\section{Those in the uncontrollable condition were told:}

Research has consistently found that most of the basic characteristics people possess are uncontrollable. What we mean by uncontrollable is that, no matter how much effort you exert, your basic characteristics will be expressed. In this way, a person who is characteristically generous with children will express their generosity: even when they try to be selfish they will still act relatively generously. In the same manner, someone who is characteristically selfish with children will express that selfishness whenever they interact with children. It may appear to them that they are acting generously, but it will still be a relatively selfish act.

Participants were then instructed that they could receive feedback about their weaknesses as well as feedback about their strengths, and were asked to write their current mailing addresses on the mailing labels they had been given. The experimenter collected the labels, and indicated that the feedback and additional questionnaires regarding its' clarity and comprehensibility would be sent to them within two business days of the experiment date.

Measures. Participants indicated their interest in receiving feedback for two social relationship domains: getting along with bosses and getting along with the elderly. The two domains were printed in random order, with weaknesses and strengths counterbalanced. Interest in feedback was indicated on a six-point scale $(1=$ not at all interested, $6=$ extremely interested $)$. After indicating their interest in each domain, participants filled out a set of questions including the controllability manipulation check (e.g., most characteristics involved in interacting with bosses (the elderly) are: $1=$ completely uncontrollable, $6=$ completely controllable), the valence manipulation check "rate the desirability of possessing the weaknesses (strengths) about which you expect to receive feedback" for both bosses and the elderly on a six-point scale $(1=$ extremely undesirable, $6=$ extremely desirable), and measures of Perceived Ability (e.g., "how would you characterize your abilities to interact with bosses (the elderly)?" $1=$ not very good, $6=$ extremely good). A median split on the average of the two perceived ability items (which were positively correlated, $r=.40$ ) was used to group participants into two (low and high) perceived ability groups. After answering the questions described above, participants were thoroughly debriefed and informed that the information they received about the feedback was fictitious.

\section{Results and discussion}

\section{Manipulation checks}

The ratings of the anticipated valence of weakness feedback across both the "bosses" and "elderly" domain were averaged, as were the ratings for strengths feedback. These two anticipated valence variables were then subjected to a 2 (Valence) $\times 2$ (Control) $\times 2$ (Perceived Ability) mixed model ANOVA, with the feedbackvalence condition as a within-participants factor. Participants ratings of weakness feedback were significantly lower than their ratings of strengths feedback $(M \mathrm{~s}=4.72$ and 5.52), $F(1,90)=53.85, p<.001$. Neither Perceived Control nor Perceived Ability affected participant's ratings of the anticipated valence of feedback.

The controllability manipulation checks were also aggregated across the "boss" and "elderly" domains within feedback valence, with the resulting "perceived control over weaknesses" and "perceived control over strengths" indices subjected to a 2 (Valence) $\times 2$ (Control $) \times 2$ (Perceived Ability) mixed model ANOVA. Results indicated that those in the controllable condition perceived significantly greater control over their abilities than those in the uncontrollable condition $(M \mathrm{~s}=4.40$ and 3.80), $F(1,90)=25.30, p<.001$. Neither feedback Valence nor Perceived Ability affected participant's ratings of their perceived control over the expression of the diagnosed attributes. Finally, ANOVAs of perceived ability in the various social relationships domain did not show any significant effect of the experimental manipulation.

\section{Main analyses}

Initial analyses of the specific domains (e.g., boss, elderly) yielded a similar pattern of results and no domain effects. Therefore, to test our primary hypotheses, participant's interest scores were averaged across both the "boss" and "elderly" domains, but within the feedback-valence condition, to create "interest in weakness" and "interest in strength" items. These items were treated as the dependent variables in a 2 (Valence) $\times 2$ (Control) $\times 2$ (Perceived Ability) mixed model ANOVA, with Valence as a repeated measure. 
The ANOVA yielded a Valence $\times$ Controllability interaction, $F(1,87)=9.09, p=.005$. As predicted, participants in the Controllable feedback condition preferred to diagnose their weaknesses relative to their strengths $(M \mathrm{~s}=4.71$ and 4.01), $t(45)=4.01, p<.01$. In contrast, participants in the Uncontrollable feedback condition were equally interested in the two types of feedback $(M \mathrm{~s}=4.55$ and 4.52). Moreover, as our theoretical analysis predicted, a 3-way Valence $\times$ Controllability $\times$ Perceived Ability interaction, $F(1,87)=4.99$, $p<.05$, showed that the effects of Valence and Controllability were conditional on participant's Perceived Ability. As can be seen in Fig. 1, participants with low perceived ability preferred to diagnose their weaknesses rather than their strengths when the diagnosed attributes were seen as controllable $(M \mathrm{~s}=4.65$ and 3.81$)$, $t(23)=5.83, p<.001$. In contrast, these participants preferred to diagnose their strengths rather than their weaknesses when the attributes were seen as uncontrollable $(M \mathrm{~s}=4.29$ and 4.00$), t(28)=2.23, p<.05$. As predicted, the feedback preferences of high perceived ability participants were not significantly affected by valence and controllability. The analysis also showed that the overall interest in feedback was greater for high than low perceived ability individuals $(M \mathrm{~s}=4.75$ and 4.19), $F(1,87)=5.85, p<.05$.

The present results support our contention that positive and negative feedback seeking depends on the controllability of the diagnosed attributes, as well as the perceived ability participants have in those areas. Specifically, the data indicate that controllability increases the desirability of negative feedback, but decreases the desirability of positive feedback. Moreover, this effect is contingent on the self-concepts of the individuals requesting feedback. As suggested by self-verification theory (Swann, 1990), participants with low perceived

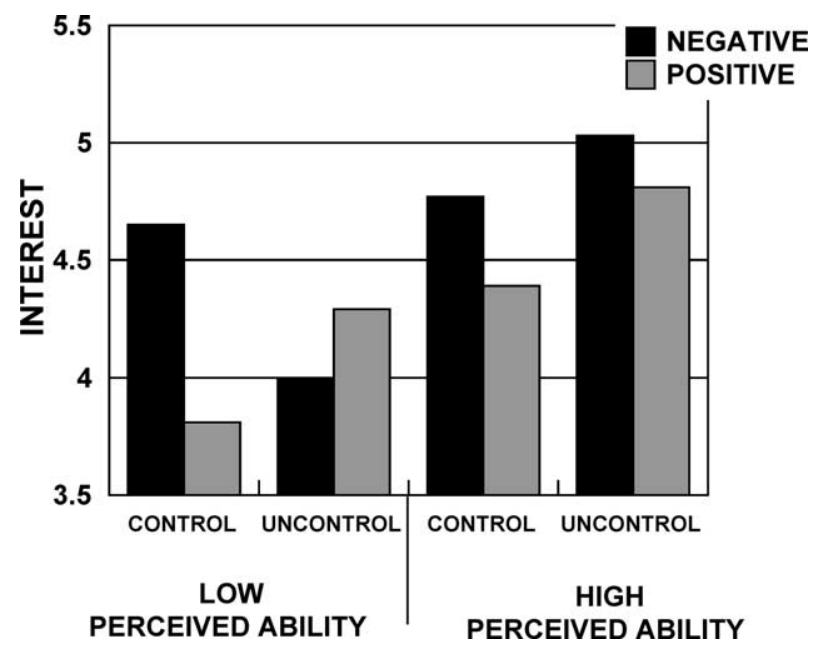

Fig. 1. Interest in feedback as a function of feedback valence, perceived controllability, and perceived ability. ability did request more negative than positive feedback. However, this occurred only when that feedback reflected controllable attributes. In contrast, when feedback was described as reflecting uncontrollable attributes, participants with low perceived abilities preferred positive feedback to negative feedback. Moreover, inconsistent with self-verification, high perceived ability participants did not prefer positive to negative feedback. Their balanced interest in the two types of feedback is consistent with the assumption that they were primarily interested in assessing their ability.

Taken together, these results suggest that people with negative self-perceptions seek out negative feedback more than positive feedback when it can be used to control their weaknesses, but avoid negative feedback in favor of positive feedback when controlling their weaknesses is not possible, in a manner more suggestive of self-enhancement (Butler, 1993; Taylor \& Lobel, 1989; Tesser, 1988; Tesser et al., 1996; Wood, 1989; Wood \& Taylor, 1991). People with positive self-perceptions showed a balanced pattern of feedback seeking, suggesting that they were primarily interested in using the feedback as an opportunity to gain better understanding of their abilities (Trope, 1986).

\section{Experiment 2}

As argued earlier, perceived control may entail not only control over the expression of an attribute, but it can also imply the ability to alter or change the attribute. Experiment 2 was designed to corroborate and extend the findings of the current research by examining this second aspect of perceived control.

Moreover, although we have theorized why perceptions of controllability and perceived ability should moderate feedback seeking, the current data do not directly assess the underlying mechanism. Thus, Experiment 2 also sought to demonstrate that the moderating roles of perceived control and perceived ability are themselves mediated by: (1) the perceived informational value or usefulness of the feedback for assessment and improvement purposes, and (2) the anticipated affective value of the self-evaluative feedback.

\section{Method}

\section{Overview}

Participants were assigned to a changeability condition, and given access either to their strengths or their weaknesses for four social relationship domains: the ability to get along with bosses, friends, neighborhood acquaintances, and the elderly. As in Experiment 1, based on self-reports of their abilities in these domains, participants were divided into a low or high perceived ability groups. Thus, Experiment 2 was a betweensubjects 2 (Strengths vs. Weakness feedback) $\times 2$ 
(Changeable vs. Unchangeable feedback) $\times 2$ (High vs. Low Perceived Ability) design.

\section{Participants}

One hundred and ninety (100 female, 90 male) college students at New York University participated in exchange for course credit. The number of participants per cell ranged from 15 to 31 .

\section{Procedure}

As in the first experiment, participants were randomly assigned to a changeability condition and handed a packet containing the instructions, the questionnaire, and an NYU mailing label. The experimental rationale was the identical to that of Study 1: Participants were offered feedback from a previously administered test of their social relationship ability in exchange for their evaluations of its comprehensibility and clarity.

Changeability manipulation. Changeability was manipulated in the second paragraph of the cover sheet, immediately following a description of the experimental rationale. In the changeable condition, participants were told:

Research using the Personality Inventory has consistently found that people can change most of their basic abilities to function in social relationships. This research has shown that through learning and experience people can change their pattern of dealing with people in various social roles. Therefore, if people try, they can develop new patterns of functioning in social relationships. There are, of course, some abilities that are less changeable. But the information we will provide you with is about those abilities that people can change through learning and experience.

Those in the unchangeable condition were told:

Research using the Personality Inventory has consistently found that people are unable to change most of their basic abilities to function in social relationships. This research has shown that despite learning and experience in dealing with others in various social roles, people are largely unable to alter the patterns of interacting with others that they learned at a very early age: their abilities tend to be fixed over time. Of course, some social relationship abilities are somewhat changeable, but the information we will provide you with is about the unchangeable abilities.

Dependent measures. The questionnaire contained separate sections, in random order, for each of the social relationship domains in which feedback could be obtained: the ability to get along with bosses, friends, acquaintances in their neighborhood, and the elderly. Each section included questions regarding how interested they were in feedback about their strengths or weaknesses in each of the four domains $(1=$ not at all interested, $6=$ extremely interested).

Following their indication of interest in feedback, participants also answered domain-specific questions about the informational value and anticipated affective value of the feedback. Two informational value items were designed to tap into the informationally oriented assessment and improvement motives. The assessment item asked participants to indicate the extent to which the information regarding their unchangeable (changeable) strengths (weaknesses) would help them develop a more accurate assessment of their ability to interact with bosses, the elderly, friends, and acquaintances $(\alpha=.76)$. The improvement item asked participants to indicate the extent to which this information would help them improve their ability to interact with bosses, the elderly, friends, and acquaintances $(\alpha=.85)$. Responses to these questions were rendered on six-point scales $(1=$ not at all, $6=$ very much).

Participants were also asked to indicate how hearing their unchangeable (changeable) weaknesses (strengths) in their ability to interact with bosses (the elderly, friends, acquaintances) would make them feel on seven adjective scales (unpleasant-pleasant, better-worse, sad-happy, bad-good, dissatisfied-satisfied, tense-relaxed, and unconfident-confident). Responses to these seven items across each social relationship domain were aggregated to form domain-specific anticipated affective value of feedback items. These four domain-specific items were then combined to form an overall anticipated affective value measure $(\alpha=.91)$.

Finally, participant's ratings of perceived ability were assessed by self-report for each domain on 6-point scales $(1=$ not very good, $6=$ extremely good). As in Experiment 1 , a median split on the average of these four ratings $(\alpha=.75)$ was used to group participants into two (low and high) perceived ability groups.

Manipulation checks. The questionnaire also included items assessing participant's perceptions of the changeability of social abilities $(1=$ completely unchangeable, $6=$ completely changeable), and the perceived valence of the feedback $(1=$ not at all desirable to possess these characteristics, $6=$ extremely desirable to possess these characteristics). After answering the questions described above, participants were thoroughly debriefed and informed that the information they received about the feedback was fictitious.

\section{Results and discussion}

\section{Manipulation checks}

The feedback valence manipulation check was analyzed in a 2 (Valence) $\times 2$ (Changeability) $\times 2$ (Perceived Ability) ANOVA. Results indicated that participant's who expected to receive feedback about their weaknesses anticipated feedback that reflected significantly less desirable attributes than those who expected to receive feedback about their strengths $(M \mathrm{~s}=4.20$ and 4.90), $F(1,182)=14.80, p<.001$. Neither Changeabil- 
ity nor Perceived Ability significantly affected the anticipated valence of feedback.

Participants' perceptions of the changeability of their abilities were examined within a 2 (Valence) $\times 2$ (Changeability) $\times 2$ (Perceived Ability) ANOVA. The manipulation of changeability was successful, with participants in the changeable feedback condition reporting significantly higher perceptions of the changeability of their abilities than those in the unchangeable feedback condition $(M \mathrm{~s}=4.66$ and 3.69$), \quad F(1,182)=44.40$, $p<.001$. This effect of the changeability manipulation was not qualified by any higher order interaction, nor did any other main effects obtain. Finally, ANOVAs of perceived ability in the various ability domains showed no effect of the experimental manipulation.

\section{Main analyses}

Feedback seeking. As in Experiment 1, initial analyses of the specific domains (e.g., boss, elderly) indicated that participant's interest in feedback did not differ as a function of domain. Therefore, participants' responses to the domain-specific interest in feedback items were averaged to form a global, univariate measure of interest in feedback. The interest in feedback measure was then analyzed in a 2 (Valence) $\times 2$ (Changeability) $\times 2$ (Perceived Ability) ANOVA.

The analysis yielded the expected Valence $\times$ Changeability interaction, $F(1,176)=25.61, p<.001$. Planned comparisons indicated that participants were more interested in receiving feedback about their weaknesses than their strengths when in the Changeable condition $(M \mathrm{~s}=4.35$ and 3.85), $t(92)=4.00, p<.01$. In contrast, when told their attributes were Unchangeable, participants were less interested in receiving feedback about their weaknesses than their strengths $(M \mathrm{~s}=4.12$ and 4.52), $t(88)=-3.09, p<.01$. However, just as in Experiment 1 , this effect was conditional on Perceived Ability, as indicated by the expected Valence $\times$ Changeability $\times$ Perceived Ability interaction, $F(1,176)=6.34$, $p<.05$ (see Fig. 2). Planned comparisons showed that participants with Low Perceived Ability sought out negative feedback more than positive feedback, but only when that feedback referred to changeable attributes $(M \mathrm{~s}=4.44$ and 3.74), $t(53)=4.38, p<.01$. In contrast, when the feedback reflected unchangeable attributes, participants with Low Perceived Ability sought out positive feedback more than negative feedback $(M \mathrm{~s}=4.32$ and 3.63), $t(51)=3.93, p<.01$. As predicted, feedback preferences by high perceived ability participants were unaffected by valence.

As in Experiment 1, the analysis also yielded a main effect of perceived ability, $F(1,176)=10.89, p<.01$, indicating higher overall interest in feedback by high than low perceived ability participants $(M \mathrm{~s}=4.34$ and 4.03$)$. However, this effect was qualified by the predicted

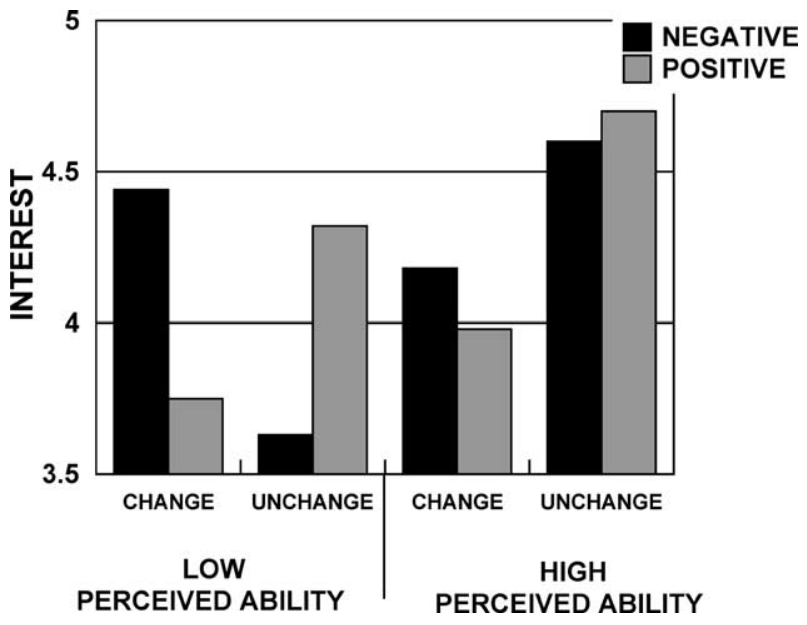

Fig. 2. Interest in feedback as a function of feedback valence, changeability, and perceived ability.

Changeability $\times$ Perceived Ability interaction, $F(1$, $176)=11.84, p<.005$, indicating a preference for unchangeable over changeable feedback by high perceived ability participants $(M \mathrm{~s}=4.59$ and 4.08$), t(74)=3.57$, $p<.01$, but not by low perceived ability participants $(M \mathrm{~s}=3.97$ and 4.09). The balanced search for positive and negative feedback regarding unchangeable skills by high perceived ability suggests that these individuals were primarily interested in assessing their ability.

We assumed that Changeability and Perceived Ability affect feedback seeking by altering the affective value and informational value of feedback. To test these assumptions, both the informational value variable and affective value variable were analyzed within separate Valence $\times$ Changeability $\times$ Perceived Ability ANOVAs.

Affective value. The analysis of affective value yielded the predicted Valence $\times$ Changeability effect, $F(1,182)=24.09, p<.001$. As hypothesized, negative feedback was more aversive than positive feedback when it was unchangeable $(M \mathrm{~s}=3.96$ and 4.46), $t(90)=5.20$, $p<.001$, but not when it was changeable $(M \mathrm{~s}=4.22$ and 3.85), $t(96)=3.10, p<.01$. The 3-way Valence $\times$ Changeability $\times$ Perceived Ability effect was also obtained, $\quad F(1,182)=6.47, \quad p<.05$. As expected, changeability made negative feedback less aversive relative to positive feedback for participants with low perceived ability but not for those with high perceived ability (see Table 1). Thus, for low perceived ability participants, negative feedback was more aversive than positive feedback when unchangeable $(M \mathrm{~s}=3.21$ and 4.03) $t(51)=4.97, p<.01$, but not when changeable $(M \mathrm{~s}=4.11$ and 3.65), $t(55)=2.84, p<.01$.

The analysis also showed a main effect of perceived ability, $F(1,182)=10.88, p<.01$. Not surprising, the feedback was expected to be more pleasing for high rather than low perceived ability participants $(M \mathrm{~s}=4.27$ and 3.98). However, this effect was qualified by the 
Table 1

Affective value as a function of feedback valence, changeability, and perceived ability

\begin{tabular}{|c|c|c|c|c|}
\hline & \multicolumn{4}{|c|}{ Affective value } \\
\hline & \multicolumn{2}{|c|}{ Low perceived ability } & \multicolumn{2}{|c|}{ High perceived ability } \\
\hline & Changeable & Unchangeable & Changeable & Unchangeable \\
\hline Negative feedback & 4.11 & 3.21 & 3.82 & 4.21 \\
\hline Positive feedback & 3.65 & 4.03 & 3.59 & 4.39 \\
\hline
\end{tabular}

predicted Changeability $\times$ Perceived Ability effect, $F(1,182)=22.94, p<.001$, indicating that participants anticipated more positive affect in response to Unchangeable feedback than changeable feedback when their perceived ability was high $(M \mathrm{~s}=4.57$ and 3.97$)$ rather than low ( $M s=3.85$ and 4.10).

Informational value: Improvement. As expected, the analysis of informational-improvement value of feedback yielded a main effect of perceived ability, indicating higher informational-improvement value of feedback for low perceived ability participants $(M=4.28)$ than high perceived ability participants $(M=3.00), F(1,180)=$ 91.63, $p<.001$. Moreover, the analysis yielded the anticipated Valence $\times$ Changeability effect, $F(1,180)=$ $24.65, p<.001$, indicating that negative feedback was more informative than positive feedback when it was changeable $(M \mathrm{~s}=3.93$ and $3.38, \quad$ respectively $)$, $t(96)=2.1, p<.05$. In contrast, positive feedback was more informative than negative feedback when the feedback was unchangeable $(M \mathrm{~s}=4.02$ and 3.24), respectively, $t(84)=2.21, p<.05$. However, the predicted Valence $\times$ Changeability $\times$ Perceived Ability effect, $F(1,180)=21.71, p<.001$, indicated that this was true only for low perceived ability participants (see Table 2 ). These participants rated negative feedback as more informative than positive feedback when it was changeable $(M \mathrm{~s}=4.86$ and 3.79), $t(55)=3.97, p<.001$, but not when it was unchangeable $(M \mathrm{~s}=3.48$ and 5.00 , respectively), $t(51)=4.87, p<.001$. For high perceived ability participants, the informational-improvement ratings of negative and positive feedback within Changeability conditions were similar.

Informational value: Assessment. As expected, the analysis of informational-assessment value of feedback yielded a main effect of changeability, indicating higher informational-assessment value of unchangeable feedback $(M=4.25)$ than changeable feedback $(M=$ 3.79), $F(1,180)=7.3, p<.01$. Moreover, the analysis yielded the anticipated Valence $\times$ Changeability effect, $F(1,180)=6.49, p<.05$, indicating that negative feedback was more informative than positive feedback when the feedback was changeable $(M \mathrm{~s}=4.42$ and 3.24 , respectively), $t(96)=5.11, p<.001$, but not when it was unchangeable $(M \mathrm{~s}=4.04$ and 4.30 , respectively $)$, $t(89)=1.1$, ns. The Changeability $\times$ Perceived Ability interaction was also significant, $F(1,180)=9.62, p<$ .01 , driven by high perceived ability participants ratings of the unchangeable feedback as more valuable for assessment purposes than the changeable feedback $(M \mathrm{~s}=4.40$ and 3.40 , respectively $), t(78)=4.02, p<$ .001. A marginal Valence $\times$ Changeability $\times$ Perceived Ability interaction, $F(1,180)=3.01, p=.08$, indicated that the Valence $\times$ Changeability interaction pattern (i.e., rating the negative feedback as more informative when it

Table 2

Informational-improvement value as a function of feedback valence, changeability, and perceived ability

\begin{tabular}{llllll}
\hline & \multicolumn{2}{l}{ Informational-improvement value } & & \multicolumn{2}{l}{ High perceived ability } \\
\cline { 2 - 3 } & \multicolumn{2}{l}{ Low perceived ability } & & & Unchangeable \\
\cline { 2 - 3 } & Changeable & Unchangeable & & Changeable & 3.00 \\
Negative feedback & 4.86 & 3.84 & 3.00 & 3.04 \\
Positive feedback & 3.79 & 5.00 & 2.96 & 3 \\
\hline
\end{tabular}

Table 3

Informational-assessment value as a function of feedback valence, changeability, and perceived ability

\begin{tabular}{llllll}
\hline & \multicolumn{2}{l}{ Informational-assessment Value } & & \\
\cline { 2 - 3 } & \multicolumn{2}{l}{ Low perceived ability } & & \multicolumn{2}{l}{ High perceived ability } \\
\cline { 2 - 3 } \cline { 5 - 6 } & Changeable & Unchangeable & & Changeable & Unchangeable \\
\hline Negative feedback & 4.57 & 3.77 & 3.75 & 4.60 \\
Positive feedback & 3.80 & 4.46 & 3.00 & 4.17 \\
\hline
\end{tabular}


pertains to changeable attributes) was true for low perceived ability participants, but not high perceived ability participants (see Table 3). Overall, then, the informational value data suggest that participants thought the negative feedback was more informative than the positive feedback for improvement and assessment when it pertained to changeable rather than unchangeable skills. This was true, however, for low perceived ability participants rather than high perceived ability participants. The latter group saw the feedback as more useful for assessment rather than improvement, particularly when it pertained to their unchangeable skills.

\section{Mediational analyses}

Although the analyses of the affective and informational value of feedback are consistent with our hypotheses, they do not tell us whether the effects of Valence, Changeability, and Perceived Ability on feedback preferences were actually mediated by the informational value and affective value of the feedback. In addition, these analyses have not yet tested the hypothesis that the informational value will operate somewhat differently for high Perceived Ability participants and low Perceived Ability participants, such that feedback preferences by those with high Perceived Ability will be more motivated by self-assessment, whereas feedback preferences by those with low Perceived Ability will be more motivated by self-improvement. If this is indeed the case, the mediating effects of informational value should be driven by the extent to which feedback facilitates increasing self-knowledge for high Perceived Ability participants, whereas the mediation of low Perceived Ability participants self-relevant information seeking should be driven by the extent to which the feedback facilitates self-improvement.

Our theoretical analysis suggests that Perceived Control will attenuate the affective impact of negative feedback, while increasing its informational value. Additionally, these effects should be greatest for those with low Perceived Ability. As indicated in the ANOVAs predicting the informational and affective value of feedback, both the affective attenuation and the informational augmentation effects of Changeability on negative feedback were supported. What remains to be demonstrated is that these effects, in turn, will lead to the increased interest in weakness feedback relative to strengths feedback, a pattern of search demonstrated across both the present experiment and Experiment 1, and that the mediational pathways will operate through the informational value of the feedback for assessment purposes for those with high Perceived Ability, and through the value of feedback for improvement for low Perceived Ability participants.

To test this mediational model, regression analyses were conducted following the steps outlined by Kenny,
Kashy, and Bolger (1997; see also Aiken \& West, 1991; Baron \& Kenny, 1986; Judd \& Kenny, 1981). To facilitate interpretation, the dichotomous predictor or initial variables "Valence," and "Changeability," were effects coded, such that negative feedback and unchangeable feedback were coded -1 , and positive feedback and changeable feedback participants were coded +1 . To tease apart the differences between high and low Perceived Ability participants without running separate analyses for each group (and thereby losing analytical power) Perceived Ability was contrast coded. Analyses focusing on Low Perceived Ability (see below) coded Low Perceived Ability as +1 (with High Perceived Ability coded as 0 ), with appropriate interaction terms constructed using these codes. Analyses focusing on High Perceived Ability used reversed codes, with new interaction terms. In addition, the informational value variable was split into two centered components, informational-assessment value and informational-improvement value, as separate mediating variables. Finally, regression analyses were conducted predicting interest in feedback. This provided the unmediated relationship (using the unstandardized coefficient $B$ ) between the "initial" or predictor variables (i.e., Valence, Changeability, Perceived Ability, and the interaction terms) and participant's interest in feedback.

Next, the centered potential mediators of anticipated affective value, informational-assessment value and informational-improvement value were treated as dependent variables in two sets of analyses to determine their path coefficients and standard errors, with the first analysis using the contrast codes that focus on low Perceived Ability participants and the second using the high Perceived Ability codes. It should be noted that although anticipated affective value, informational-assessment value and informational-improvement value were measured after interest in feedback to protect the primary DV (interest in feedback), our theory suggests that these value calculations occur prior to the decision to seek or avoid feedback.

Finally, two analyses were conducted to better understand the mediation of the 3-way Valence $x$ Change $\times$ Perceived Ability interaction effect on interest in feedback. Both analyses regressed the initial variables and the mediating variables simultaneously on interest in feedback, and indirect effects tests were conducted on each mediator that was significantly predicted by initial variables that predicted feedback interest in the unmediated model.

\section{Low perceived ability mediation}

Results from the first analysis suggested that, although all three potential mediators were significantly predicted by multiple initial variables, low Perceived Ability participants' feedback seeking was motivated primarily by improvement and affective motives. 
Informational-improvement value significantly predicted interest in feedback ( $\left.B_{\text {improve }}=.33, p<.01\right)$, whereas informational-assessment value did not. Moreover, as our pragmatic framework also suggests, the anticipated affective value of feedback was a significant predictor of interest in feedback $\left(B_{\text {affect }}=.25, p<.05\right)$. Taken together, the positive coefficients between informational-improvement value and interest in feedback and affective value and interest in feedback provide evidence for the underlying competition between informational and affective motives, particularly under conditions where the offered feedback is negative (see Fig. 3).

Indirect effects tests indicated that both informational-improvement value and affective value were significant mediators of the Valence $\times$ Change interaction. Not only was the direct path between the Valence $x$ Change interaction and feedback interest from the initial regression partially mediated, but the indirect effects tests indicated that the reduction was accounted for by both the informational-improvement mediator and the affective value mediator $\left(Z_{\text {improvement }}=-2.47\right.$, $p<.01$, and $Z_{\text {affect }}=-2.19, p<.01$, respectively). Taken together, these results suggest that the primary informational self-evaluative motive for low Perceived Ability participants was self-improvement. At the same time, participants were also sensitive to the affective implications of the feedback, providing strong support for the contention that these motives not only co-exist, but that they are potential sources of self-regulatory conflict.

\section{Low Perceived Ability}

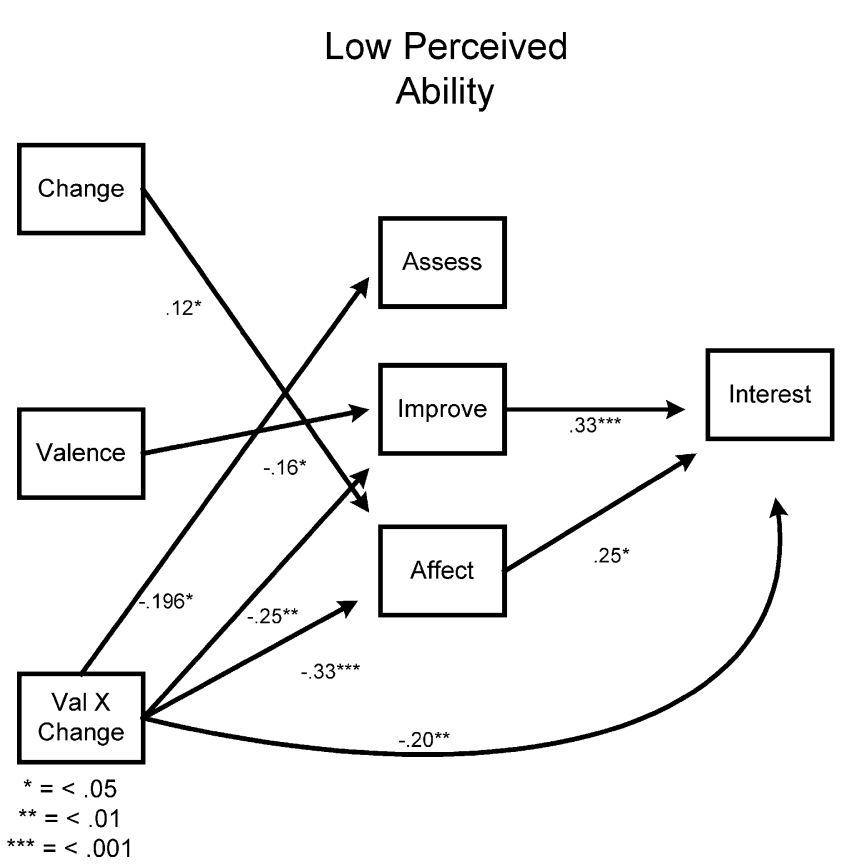

It should be noted that although informational-assessment value did not mediate any of the initial effects, it was significantly predicted by the Valence $\times$ Perceived Ability interaction $(B=-.32, p<.01)$. This suggests that positive feedback was seen as less useful for assessment purposes by those with low Perceived Abilities.

It is also important to note that Perceived Ability also significantly predicted the improvement value of feedback $(B=3.11, p<.01)$, despite the fact that Perceived Ability did not have a direct effect on interest in feedback as an unmediated, initial variable. It seems, then, that low Perceived Ability participants found the feedback to be more useful for improvement than high Perceived Ability participants, suggesting that improvement may well be a relatively strong motive for those with low Perceived Abilities (as compared to those with high Perceived Abilities).

\section{High perceived ability mediation}

The mediation effects were very different for high Perceived Ability participants. Unlike the low Perceived Ability participants, the high Perceived Ability participants' information seeking was mediated by the informational-assessment value of feedback, as opposed to the informational-improvement value $(B=.15, p<.05)$. Additionally, affective value had only a marginal impact on interest in feedback $(B=.20, p=.06)$, suggesting that the importance of affective value is attenuated for participants with high perceived ability.

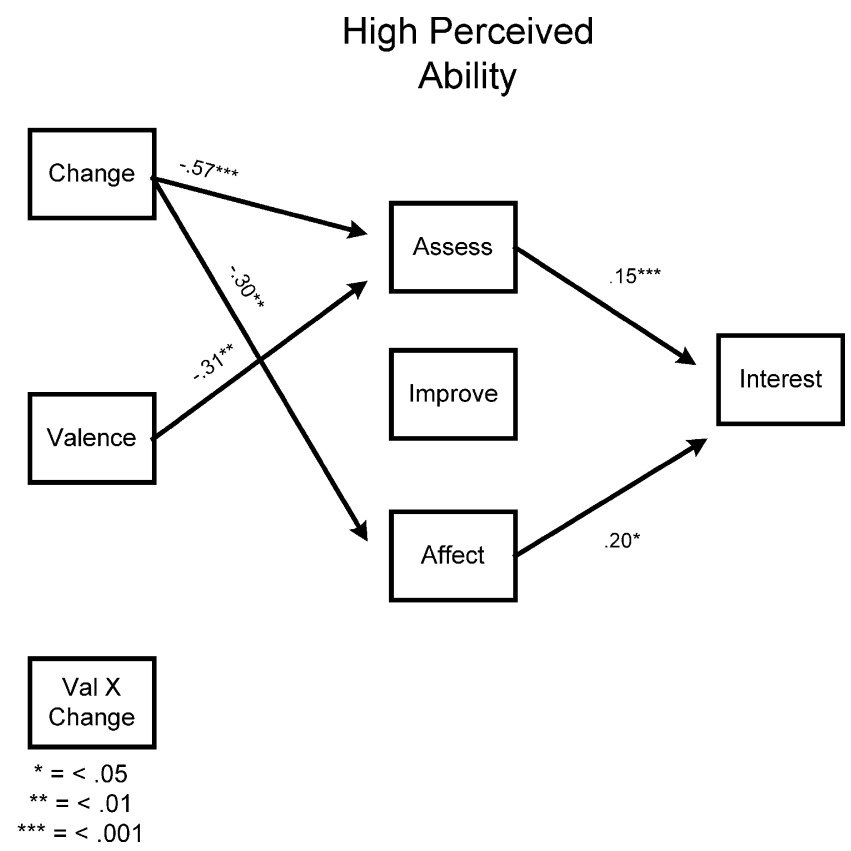

Fig. 3. Interest in feedback as a function of feedback valence, changeability, and perceived ability, mediated by informational and affective value. All initial variables are effects coded, with negative, unchangeable, and low perceived ability conditions coded -1 , and positive, changeable, and high perceived ability conditions coded +1 . 
As the only significant indirect path between significant initial variables and the mediating variables was the Changeability $\rightarrow$ Informational-Assessment Value $\rightarrow$ Interest path, indirect effects tests were conducted only for this path. The indirect effects test indicated that the initial Changeability main effect was significantly mediated by informational-assessment value $\left(Z_{\text {assess }}=\right.$ $-2.346, p<.01)$. This suggests that self-assessment was the primary motive underlying self-evaluative feedback seeking for high Perceived Ability participants.

Taken together, the present results support our contention that willingness to receive negative feedback depends on one's perceived ability and the perceived changeability of the ability. The data suggest that individuals will seek negative (rather than positive) feedback when their perceived ability is low (rather than high) and the feedback refers to changeable (rather than unchangeable) weaknesses. Moreover, as indicated by the mediational analyses, this is likely to be the case because, for individuals with low perceived ability, the belief that weaknesses are changeable diminishes the negative affect impact of the feedback, while enhancing its informational value, particularly with regards to improvability.

Of course, a preference for negative feedback by individuals with low perceived ability is consistent with self-verification theory (see Swann, 1990). However, consistent with Experiment 1, the present experiment shows that this preference holds true only when the feedback diagnoses changeable abilities. When the abilities are construed as unchangeable, individuals with low (rather than high) perceived ability prefer positive to negative feedback. This finding, in conjunction with the mediation analysis, suggests that in seeking negative feedback, low perceived ability individuals were trying to learn how to improve themselves rather than to protect their pre-existing self beliefs. Moreover, in order to find support for self-verification in the current analyses, there should have been significant main effects of feedback valence in each of the two sets of regressions, such that low Perceived Ability participants should have preferred negative feedback, and high Perceived Ability participants should have preferred positive feedback. As no such effects were obtained, the current analyses provide evidence for the use of negative feedback for improvement by low Perceived Ability participants, rather than self-verification.

\section{General discussion}

The goal of the present research was to investigate how perceived control over one's abilities and one's beliefs regarding one's standing on these abilities affect feedback seeking. Based on an analysis of the informational and affective value of feedback, we hypothe- sized that individuals would seek negative (rather than positive) feedback to the extent that: (a) the feedback diagnoses controllable or changeable abilities and (b) the individuals' perceived level of ability is low. The results provide strong converging evidence for our hypotheses. Overall, participants were more interested in negative rather than positive feedback when the feedback diagnosed a controllable or changeable ability. When the feedback diagnosed an uncontrollable or unchangeable ability, participants actually preferred positive to negative feedback. Moreover, this effect of perceived control on interest in negative rather than positive feedback held true only for participants with low perceived ability. High perceived ability participants were equally interested in positive and negative feedback regardless of perceived control over the diagnosed attributes.

These results are consistent with the present pragmatic analysis of feedback seeking. According to this analysis, feedback seeking depends on the informational and affective value of the feedback. In the case of negative feedback, informational value comes at the expense of affective value. On the one hand, this feedback can be used for learning purposes, namely, for diagnosing one's weaknesses and finding ways in which they can be overcome through self-improvement (Butler, 1993; Taylor et al., 1995; Trope, 1986; Trope \& Neter, 1994). On the other hand, by uncovering one's weaknesses, negative feedback threatens one's self-esteem and is therefore emotionally aversive (Brown, 1990; Sedikedes \& Strube, 1997; Tesser et al., 1996; Wood, 1989). Situations that offer negative feedback thus pose a conflict between pursuing learning goals and defensive goals (see Butler, 1993; Dweck \& Leggett, 1988).

We proposed that the relative strength of affective and informational concerns depends on perceived control over one's abilities. More specifically, perceived control should increase the informational value of negative feedback, while decreasing its negative affective value. Such feedback can tell individuals what abilities they need to improve, and knowing that these abilities are actually improvable may diminish the emotional threat posed by the feedback. The present pragmatic analysis further suggests that these effects of perceived control should obtain to the extent that individuals think their abilities in the diagnosed domain are deficient. For these individuals, self-improvement should be particularly important. As a result, diagnosis of weaknesses that can be controlled or modified is likely to be perceived as very useful. Moreover, the diagnosis of uncontrollable or unchangeable weaknesses may be particularly aversive to low perceived ability individuals because such diagnosis implies that their abilities will always remain deficient and that the negative consequences of these deficiencies are unavoidable. Thus, 
both informational and affective considerations make perceived control particularly valuable to individuals with low perceived abilities. When these individuals think that they can change or control themselves, their pursuit of learning, mastery-related goals does not come at the expense of ego-defensive, esteem-related concerns (Butler, 1993; Dweck \& Leggett, 1988; Elliot \& Harackiewicz, 1996).

Consistent with our assumption that changeability and controllability are two aspects of perceived control, our findings show that, overall, feedback seeking was similarly affected by these two variables. Our findings also suggest an interesting difference between changeability and controllability. When perceived ability was low, there was no overall preference for changeable over unchangeable feedback or for controllable over uncontrollable feedback. However, when perceived ability was high, unchangeable feedback was preferred to changeable feedback (Study 2), but uncontrollable feedback was not preferred to controllable feedback (Study 1). We argued that for high perceived ability individuals feedback about unchangeable (rather than changeable) skills is valuable because it allows them to assess the stable aspects of their ability and, thereby, better predict performance outcomes and make more informed task choices. For example, by knowing one's social skills, one can better predict the outcome of social situations and better choose such situations. Controllability of the diagnosed skills is less important to high perceived ability individuals because usually there are no obvious advantages in controlling desirable skills. Social skills, for example, are qualities people want to use rather than suppress. Our high perceived ability participants interest in feedback was therefore unaffected by the controllability of the diagnosed skills.

The present finding that perceived control over one's abilities increases interest in negative feedback is consistent with Dunning's (1995) research. Moreover, Dunning's finding that such interest in negative feedback is particularly true when the assessed ability is important is consistent with the present pragmatic view of feedback seeking (see also Trope \& Pomerantz, 1998). However, in Dunning's study, interest in negative feedback was inferred from participants' interest in feedback in a domain in which they initially failed. Feedback seeking could, therefore, be due to participants' low perceived ability following initial failure, the new negative feedback they expected to receive, or both. The present studies allow us to separate the influence of the two factors on feedback seeking by offering participants with pre-existing low or high perceived ability both positive and negative feedback and showing that the interest in negative feedback (regarding controllable abilities) holds for participants with low rather than high perceived ability.
The tendency for individuals with negative self views to seek negative self-relevant information has been interpreted as an attempt to defend pre-existing self-beliefs (see Swann, 1990). Defending such beliefs may sometimes play an important role in self-evaluation (see Pelham, 1991; Pelham \& Swann, 1994; Sedikedes, 1993; Sedikedes \& Strube, 1995, 1997; Swann \& Schroeder, 1995; Tesser et al., 1996). However, the present research suggests that the interest in negative feedback by individuals with negative self views may be motivated, at least in part, by more pragmatic, self-improvement concerns. Indeed, when negative feedback could not be used for self-improvement purposes (that is, when it pertained to uncontrollable or unchangeable attributes), participants with low perceived ability strongly preferred positive feedback. Protection of pre-existing selfbeliefs also predicts a preference for positive feedback by individuals with positive self views. The present findings do not support this prediction. High perceived ability participants showed balanced search for positive and negative information regarding their stable skills, suggesting that these participants were primarily interested in self-assessment, namely, achieving better understanding and prediction of their future performance in the diagnosed ability domain.

\section{Conclusion}

Overall, the results of the present studies paint a picture of people as pragmatic self-evaluators, flexibly taking into account various motivational considerations in deciding how to select self-relevant information (see Butler, 1993; Trope \& Fishbach, 2000; Trope \& Liberman, 1996; Trope \& Neter, 1994). When individuals think their abilities are deficient and, therefore, need to be improved, they tend to be sensitive to what the feedback diagnoses (weaknesses or strengths) and to the controllability or changeability of the diagnosed abilities. When the feedback diagnoses controllable abilities, these individuals will seek negative feedback because such feedback is informationally useful and emotionally tolerable. However, when the feedback diagnoses uncontrollable abilities, the same individuals will avoid negative feedback because the feedback becomes less informationally useful and more emotionally aversive. These findings have important implications for real life social, academic, and vocational situations. People's general beliefs about the fixed nature of ability and their concern with protecting their self-esteem may prevent them from attempting to improve themselves (see Butler, 1993; Dweck \& Leggett, 1988; Elliot \& Harackiewicz, 1996). The present research suggests that framing specific skills as controllable may help overcome some of the obstacles to self-improvement by facilitating openness to information about one's deficiencies. 


\section{References}

Abramson, L. Y., Metalsky, G. I., \& Alloy, L. B. (1989). Hopelessness depression: A theory-based subtype of depression. Psychological Review, 96, 358-372.

Abramson, L. Y., Seligman, M. E. P., \& Teasdale, J. D. (1978) Learned helplessness in humans: Critique and reformulation. Journal of Abnormal Psychology, 87, 49-74.

Aiken, L. S., \& West, S. G. (1991). Multiple regression: Testing and interpreting interactions. Newbury Park, CA: Sage.

Alicke, M. D. (1985). Global self-evaluation as determined by the desirability and controllability of trait adjectives. Journal of Personality and Social Psychology, 59, 1621-1630.

Baron, R. M., \& Kenny, D. A. (1986). The moderator-mediator variable distinction in social psychological research: Conceptual, strategic and statistical considerations. Journal of Personality and Social Psychology, 51, 1173-1182.

Brown, J. D. (1990). Evaluating one's abilities: Shortcuts and stumbling blocks on the road to self-knowledge. Journal of Personality and Social Psychology, 50, 149-167.

Brown, J. D., \& Dutton, K. A. (1995). Truth and consequences: The costs and benefits of accurate self-knowledge. Personality and Social Psychology Bulletin, 21, 1288-1296.

Butler, R. (1993). Effects of task- and ego-achievement goals on information seeking during task engagement. Journal of Personality and Social Psychology, 65, 18-31.

Dunning, D. (1995). Trait importance and modifiability as factors influencing self-assessment and self-enhancement motives. Personality and Social Psychology Bulletin, 21, 1297-1306.

Dweck, C. A. (1991). Self-theories and goals: Their role in motivation, personality and development. In R. Dienstbeir (Ed.), Nebraska Symposium on Motivation, 1990 (pp. 199-235). Lincoln: University of Nebraska Press.

Dweck, C. A., Hong, Y. Y., \& Chiu, C. Y. (1993). Implicit theories: Individual differences in the likelihood and meaning of dispositional inference. Personality and Social Psychology Bulletin, 19, 644-656.

Dweck, C. A., \& Leggett, E. L. (1988). A social-cognitive approach to motivation and personality. Psychological Review, 95, 256-273.

Elliot, A. J., \& Harackiewicz, J. M. (1996). Approach and avoidance achievement goals and intrinsic motivation: A mediational analysis. Journal of Personality and Social-Psychology, 70, 461-475.

Judd, C. M., \& Kenny, D. A. (1981). Process analysis: Estimating mediation in treatment evaluations. Evaluation Review, 5, 602-619.

Kenny, D. A., Kashy, D. A., \& Bolger, N. (1997). Data analysis in social psychology. In D. Gilbert, S. T. Fiske, \& G. Lindzey (Eds.), Handbook of social psychology (4th ed.). New York: McGraw-Hill.

Pelham, B. W. (1991). On confidence and consequence: The certainty and importance of self-knowledge. Journal of Personality and Social Psychology, 60, 518-530.

Pelham, B. W., \& Swann, W. B., Jr. (1994). The juncture of intrapersonal and interpersonal knowledge: Self-certainty and interpersonal congruence. Personality and Social Psychology Bulletin, 20, 349-357.

Pyszczynski, T. A., \& Greenberg, J. (1987). Toward an integration of cognitive and motivational perspectives in social inference: A biased hypothesis-testing model. In L. Berkowitz (Ed.), Advances in experimental social psychology (Vol. 20, pp. 297-334). New York: Academic Press.

Ruble, D. N., \& Frey, K. S. (1991). Changing patterns of behavior as skills are acquired: A functional model of self-evaluation. In J. Suls, \& T. A. Wills (Eds.), Social comparison: Contemporary theory and research (pp. 79-113). Hillsdale, NJ: Erlbaum.

Sedikedes, C. (1993). Assessment, enhancement and verification determinants of the self-evaluation process. Journal of Personality and Social Psychology, 65, 317-338.
Sedikedes, C., \& Strube, M. J. (1995). The multiply motivated self. Personality and Social Psychology Bulletin, 21, 1330-1335.

Sedikedes, C., \& Strube, M. J. (1997). Self evaluation: To thine own self be good, to thine own self be sure, to thine own self be true, and to thine own self be better. In M. P. Zanna (Ed.), Advances in experimental social psychology (Vol. 30, pp. 209-251). New York: Academic Press.

Steele, C. M. (1988). The psychology of self-affirmation: Sustaining the integrity of the self. In L. Berkowitz (Ed.), Advances in experimental social psychology (Vol. 21, pp. 261-302). New York: Academic Press.

Swann, W. B., Jr (1990). To be adored or to be known? The interplay of self-enhancement and self-verification. In R. M. Sorrentino, \& E. T. Higgins (Eds.), Handbook of motivation and cognition: Foundations of social behavior (Vol. 2, pp. 408-448). New York: Guilford

Swann, W. B., Jr., \& Schroeder, D. G. (1995). The search for beauty and truth: A framework for understanding reactions to evaluations. Personality and Social Psychology Bulletin, 21, 13071318.

Taylor, S. E., \& Lobel, M. (1989). Social comparison activity under threat: Downward evaluation and upward contacts. Psychological Review, 96, 569-575.

Taylor, S. E., Neter, E., \& Wayment, H. A. (1995). Self-evaluation processes. Personality and Social Psychology Bulletin, 21, 12781287.

Tesser, A. (1988). Toward a self-evaluation maintenance model of social behavior. In L. Berkowitz (Ed.), Advances in experimental social psychology (Vol. 21, pp. 181-227). New York: Academic Press.

Tesser, A., Martin, L. L., \& Cornell, D. P. (1996). On the substitutability of self-protective mechanisms. In P. M. Gollwitzer, \& J. A. Bargh (Eds.), The psychology of action: Linking cognition and motivation to behavior (pp. 48-68). New York: Guilford.

Trope, Y. (1975). Seeking information about one's own ability as a determinant of choice among tasks. Journal of Personality and Social Psychology, 32, 1004-1013.

Trope, Y. (1983). Self-assessment in achievement behavior. In J. Suls, \& A. G. Greenwald (Eds.), Psychological perspectives on the self (Vol. 2). Hillsdale, NJ: Erlbaum.

Trope, Y. (1986). Self-assessment and self-enhancement in achievement motivation. In R. M. Sorrentino, \& E. T. Higgins (Eds.), Handbook of motivation and cognition: Foundations of social behavior (Vol. 1, pp. 350-378). New York: Guilford.

Trope, Y., \& Fishbach, A. (2000). Counteractive self-control processes in overcoming temptation. Journal of Personality and Social Psychology.

Trope, Y., \& Liberman, A. (1996). Social hypothesis testing: Cognitive and motivational mechanisms. In E. T. Higgins, \& A. W. Kruglanski (Eds.), Social psychology: Handbook of basic principles (pp. 239-270). New York: Guilford.

Trope, Y., \& Neter, E. (1994). Reconciling competing motives in selfevaluation: The role of self-control in feedback seeking. Journal of Personality and Social Psychology, 66, 646-657.

Trope, Y., \& Pomerantz, E. (1998). Resolving conflicts among selfevaluative motives: The role of positive experiences in overcoming defensiveness. Motivation \& Emotion, 22, 53-72.

Weiner, B. (1986). An attributional theory of motivation and emotion. New York: Springer.

Wood, J. V. (1989). Downward comparison principals in social psychology. Psychological Bulletin, 90, 245-271.

Wood, J. V., \& Taylor, K. L. (1991). Serving self-relevant goals through social comparison. In J. Suls, \& T. A. Wills (Eds.), Social comparison: Contemporary theory and research (pp. 23-49). Hillsdale, NJ: Erlbaum. 